\title{
Vorwort des Gastherausgebers
}

\author{
Robert Galler \\ Leoben, Österreich \\ Online publiziert 29. November 2016
}

Liebe Geotechniker und Freunde des konstruktiven Tiefbaus!

Die Stabilitätsfrage von Hängen und Böschungen gehört zu den grundlegenden Fragestellungen in unserem Aufgabengebiet. Bevor mit der Errichtung eines Untertagebauwerks begonnen werden kann, ist nahezu immer ein Voreinschnitt herzustellen und im Zuge dessen auch Hangsicherung einzubringen. Um eine zielgerichtete Planung durchführen zu können, müssen die bodenmechanischen und erdstatischen Grundlagen, die zeigen, warum es überhaupt zu einer Rutschung kommen kann, verstanden werden.

Befindet sich ein Voreinschnitt bereits im Bauzustand und stellt sich dabei heraus, dass die anstehenden geotechnischen Gegebenheiten nicht jenen der geologischen Prognose, die der Planung zugrunde gelegt waren, entsprechen, benötigen wir Verfahren, die eventuell entstehende Hangbewegungen rechtzeitig im Vorfeld erkennen lassen.

Werden entstehende Hangbewegungen im Vorfeld erkannt, müssen Maßnahmen bereitgestellt werden, die imstande sind, Rutschungen zu verhindern. Im Fall dessen, dass die Hangbewegungen bereits eingetreten sind braucht es entsprechende Maßnahmen zur Sanierung.

Um all diese Fragestellungen im Expertenkreis zu diskutieren, lud der Lehrstuhl für Subsurface Engineering gemeinsam mit der VÖBU am 17. November 2016 zu einem Spezialseminar mit dem Titel Stabilitätsfragen in der Geotechnik in die AULA der Montanuniversität Leoben ein. Auftraggeber- und Baubehördenvertreter, Mitarbeiter/ innen von entsprechenden Planungsbüros und Bauunternehmen sowie Mitarbeiter der Universitäten folgten dieser Einladung und konnten einen regen Erfahrungsaustausch genießen.

Das vorliegende Heft der BHM versucht die Beiträge dieses Spezialseminars möglichst vollständig wiederzugeben.

R. Galler ( $₫)$

Leoben, Österreich

robert.galler@unileoben.ac.at
Erweitert wird dieses Spektrum der Beiträge noch durch einen Forschungsbeitrag für den maschinellen Tunnelbau, in welchem neue Messtechnologien für Tunnelbohrmaschinen und ihr Nutzen diskutiert werden.

In eigener Sache darf ich mich in dieser Ausgabe der BHM ganz herzlich bei all jenen bedanken, die es ermöglicht haben, das Forschungs-, Entwicklungs-, Trainingsund Ausbildungszentrum research@ZaB - Zentrum am Berg Wirklichkeit werden zu lassen. Der feierliche Tunnelanschlag konnte in Gegenwart höchstrangiger Vertreter aus Politik und Wirtschaft sowie der Eisenerzer Bevölkerung gefeiert werden.

Ferner möchte ich mich auch bei all jenen bedanken, die mich auf dem Weg zum Österreicher des Jahres tatkräftig unterstützt haben und damit gleichzeitig die Montanuniversität Leoben ins Zentrum des Geschehens in Sachen Forschung rücken haben lassen.

Ich wünsche Ihnen eine frohe Weihnachtszeit und Alles Gute für das Neue Jahr 2017!

Ein herzliches Glückauf!

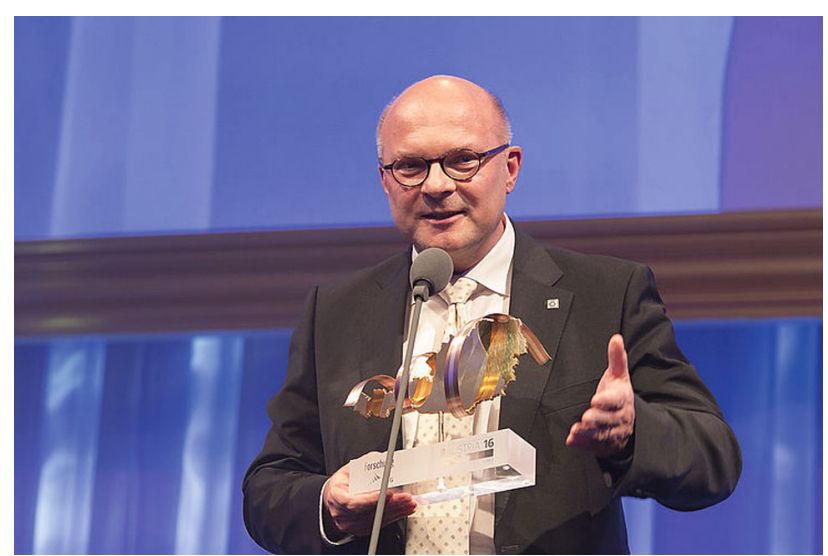

Univ.-Prof. Dr. Robert Galler bei der Preisverleihung als „Österreicher des Jahres“ (Fotocredit: Mirjam Reither) 